\title{
Outcome and Cost Effectiveness of Ultrasonographically Guided Surgical Clip Placement for Tumor Localization in Patients undergoing Neo-adjuvant Chemotherapy for Breast Cancer
}

\author{
Imrana Masroor ${ }^{1 *}$, Sana Zeeshan ${ }^{2}$, Shaista Afzal' ${ }^{1}$, Saira Naz Sufian", Madeeha \\ Ali $^{2}$, Shaista Khan ${ }^{2}$, Khabir Ahmad ${ }^{2}$
}

\begin{abstract}
Background: To determine the outcome and cost saving by placing ultrasound guided surgical clips for tumor localization in patients undergoing neo-adjuvant chemotherapy for breast cancer. Materials and Methods: This retrospective cross sectional analytical study was conducted at the Department of Diagnostic Radiology,Aga Khan University Hospital, Karachi, Pakistan from January to December 2014. A sample of 25 women fulfilling our selection criteria was taken. All patients came to our department for ultrasound guided core biopsy of suspicious breast lesions and clip placement in the index lesion prior to neo-adjuvant chemotherapy. All the selected patients had biopsy proven breast cancer. Results: The mean age was $45 \pm 11.6$ years. There were no complications seen after clip placement in terms of clip migration or hemorrhage. The cost of commercially available markers was approximately PKR 9,000 (US\$ 90) and that of the surgical clip was PKR 900 (US\$ 9). The cost of surgical clips in 25 patients was PKR 22,500 (US\$ 225), when compared to the commercially available markers which may have incurred a cost of PKR 225,000 (US\$ 2,250). The total cost saving for 25 patients was PKR 202,500 (US\$ 2, 025), making it PKR 8100 (US\$ 81) per patient. Conclusions: The results of our study show that ultrasound guided surgical clip placement in index lesions prior to neo-adjuvant therapy is a safe and cost effective method to identify tumor bed and response to treatment for further management.
\end{abstract}

Keywords: Breast cancer - neo adjuvant chemotherapy - biopsy - surgical clip marker

Asian Pac J Cancer Prev, 16 (18), 8339-8343

\section{Introduction}

Neo-adjuvant chemotherapy (NAC) has revolutionized the care for patients with operable and inoperable breast cancers. It has helped to reduce the mortality and improve the surgical options in terms of making inoperable tumors excisable and conversion to breast conservation in operable breasts and early collection of information on the treatment response and tumor biology of breast cancer (Kaufmann et al., 2007; Youn et al., 2015). Published studies have demonstrated a significant clinical response rate of the primary tumor to neo-adjuvant chemotherapy with success rates of $80 \%$ to $90 \%$ (Abdel-Razeq et al., 2011; Lobbes et al., 2013). At times, a complete clinical, radiological and pathological response can be achieved after neoadjuvant therapy, but the results vary widely (6-33\%) (Abdel-Razeq et al., 2011; Lobbes et al., 2013). Although a pathological complete response is aimed and is associated with good prognosis, a complete clinical and radiological response is associated with difficulty for breast surgeon to identify the exact area for excision corresponding to primary tumor bed. To avoid this complication, use of radiopaque markers for the identification of primary tumors after neo-adjuvant chemotherapy has long been used worldwide and has been reported as a safe technique for localization of tumor bed for surgical excision after neo-adjuvant chemotherapy, particularly with a complete clinical and radiological response (Oh ., 2007; Kaufmann et al., 2012; Youn et al., 2015). In a study by (Dash et al., 1999) all the patients $(n=28)$ underwent clip placement and preoperative chemotherapy and the study concluded that in $35.7 \%$ of patients, it would have been impossible and in $21.4 \%$ difficult to do wire localizations without clip placement. They reported that clip placement prior to neo-adjuvant chemotherapy was valuable in $57 \%$ of patients at the time of needle localization.

In another study (Edeiken et al., 1999), the metallic markers were the only remaining evidence of original tumor site in $47 \%$ (23 out of 49 ) of patients. In this study, all metallic markers were placed under ultrasound guidance in an assumption that tumor will show complete or near complete response to neo-adjuvant therapy. This 
study reported that radio opaque marker placement prior to neo-adjuvant chemotherapy addresses the problem of preoperative tumor bed localization in patients who are expected to achieve a complete or near complete response.

In most parts of the world, commercially available titanium based clips are used for tumor bed localization. These are placed within the tumor with the help of radiological assistance such as ultrasound and mammography (Margolin et al., 2003). Many other studies conducted in west support the use of radiopaque clip placement preoperatively for tumor bed localization before neo-adjuvant chemotherapy. The cost of such clips varies from between PKR 9,000 to 15,000 (US\$ 90 to 150) depending upon make and visibility options. Use of such radiopaque markers in patients belonging to third world countries, where the entire treatment expenses are borne by the patients, can add a substantial cost burden. Therefore, instead of using the expensive commercially available markers, we utilized the standard titanium surgical clips, also known as ligaclips used in various breast surgeries, as our radiopaque marker. These clips can be placed easily by a $16 \mathrm{G}$ spinal needle, without the need for commercially available applicator. These clips are easily available, cost effective (PKR 900, US\$ 9), Magnetic Resonance Imaging (MRI) compatible and no complications have been reported after their use. Therefore, the purpose of this study was to review the cost effectiveness of these surgical clips in terms of tumor bed localization and any possible complications encountered during or after clip placement, such as clip migration, bleeding, infection and pain.

\section{Materials and Methods}

It was a retrospective analytical study, conducted at radiology department of Aga Khan University hospital. The study duration was from January to December 2014. Convenience sampling was used. Exemption was granted from Ethical Review Committee of Aga Khan University Hospital (ERC \# 3594-Rad-ERC-15).

\section{Informed consent}

All patients had consented to the placement of ligaclip within the tumor bed after core biopsy and had signed the pre-designed hospital consent form. They were counseled in detail by the primary surgeon as well as the radiologist before the performance of procedure about the nature of ligaclip, the method and purpose of placement and the rare possibility of clip migration as well as associated complications.

\section{Inclusion criteria}

All female patients who had a histopathological diagnosis of breast cancer on core needle biopsy and were to undergo neo-adjuvant chemotherapy for reduction in tumor size were included in the study.

All patients who had their mammograms, ultrasound and core biopsy and guided clip placement followed by surgery at our institution were included in the study.

\section{Exclusion criteria}

Patients with incomplete medical records or who had surgery outside our institution were excluded.

Patients with Stage IV disease and those who showed progression of disease during or after chemotherapy were also excluded.

\section{Clip placement technique}

All breast biopsies and subsequent clip placements were performed by radiologist with 5 to 10 year experience in breast imaging using ultrasound guidance.

All patients initially underwent ultrasound guided core biopsy of index lesion using a $14 \mathrm{G}$ needle with an automated gun, at least 4 to 5 cores were taken (Figure 1). This was followed by placement of a surgical clip in the index lesion using a $16 \mathrm{G}$ spinal needle. The surgical clip was closed by an applicator for its easy passage through the bore of $16 \mathrm{G}$ needle. The stylet of $16 \mathrm{G}$ spinal needle was used to advance the clip through the bore of spinal needle into the center of lesion using ultrasound guidance. Following successful placement of clips, two mammographic views were performed in craniocaudal (Figure 2) and mediolateral projection to ascertain the correct position of clip within the lesion before discharging the patient.

Following neo-adjuvant chemotherapy, the mammograms were repeated to see the response of the index lesion (Figure 3). In case of complete clinical and radiological response, if breast conservation was to be performed, the patient underwent bracketing (Figure 4) or needle localization of surgical clip (Figure 5) under mammographic guidance prior to definitive surgery. The excised specimen was subjected to specimen radiography to ascertain that the targeted area has been removed with satisfaction (Figure 6). In case of mastectomy, no bracketing or needle localization was performed.

All mammogram and ultrasound images were reviewed by two experienced radiologists with 5 to 10 years of experience in breast imaging. All images of pre and post ligaclip placement and post neo-adjuvant chemotherapy were reviewed for correct clip placement, and possible complications such as clip migration. Medical record files of all patients were also seen for any possible complaints after ligaclip insertion on their follow up visit in breast clinic.

\section{Data analysis}

Information was extracted retrospectively from the hospital's computerized database and analysis was done using SPSS version 20. Frequencies and means were calculated. The percentage of complications was also recorded. The cost effectiveness was determined by calculating the cost of ligaclip and commercially available metallic markers for 25 patients in Pakistani rupees and US dollars, the cost was also determined for individual patient. The two figures were subtracted to get the cost saving.

\section{Results}

From January 2014 to December 2014, 350 patients underwent ultrasound guided core biopsy of breast 
lesions at our institute. Out of these 192 biopsies were malignant and 158 were benign on histopathology. All patients with bilateral breast procedures were taken as $n=$ 2. A total of 192 cases had ligaclips placed within their lesions at the time of core biopsy. Out of 192, only 25 patients fulfilled our selection criteria i.e. biopsy proven malignant disease of breast with ligaclip in index lesion, non-metastatic disease, having received NAC followed by surgery (mastectomy or breast conservation) and available pre and post neo-adjuvant mammograms with ligaclip visible in them. Ligaclip in all cases were placed with a uniform method prior to initiation of NAC. All patients with benign lesions, incomplete records and progression of disease during or after chemotherapy were excluded from this study. The procedures of ligaclip placement as well as definitive surgery for all the included cases were performed at our institute only. The mean age of patients was $45 \pm 11$.6years. One to 3 ligaclips were placed in a single lesion. The duration of chemotherapy was from 75 to 200 days. Out of 25 lesions, 15 were in right breast and 10 in the left breast. Maximum number $(n=10)$ of lesions were in upper outer quadrant. Out of 25 clips 23 were within the index lesion, two of them were 1 to $2 \mathrm{~mm}$ anterior to the lesion. There was no migration of clips on post procedure mammograms and subsequent mammograms post chemotherapy, preoperative mammogram and on surgical

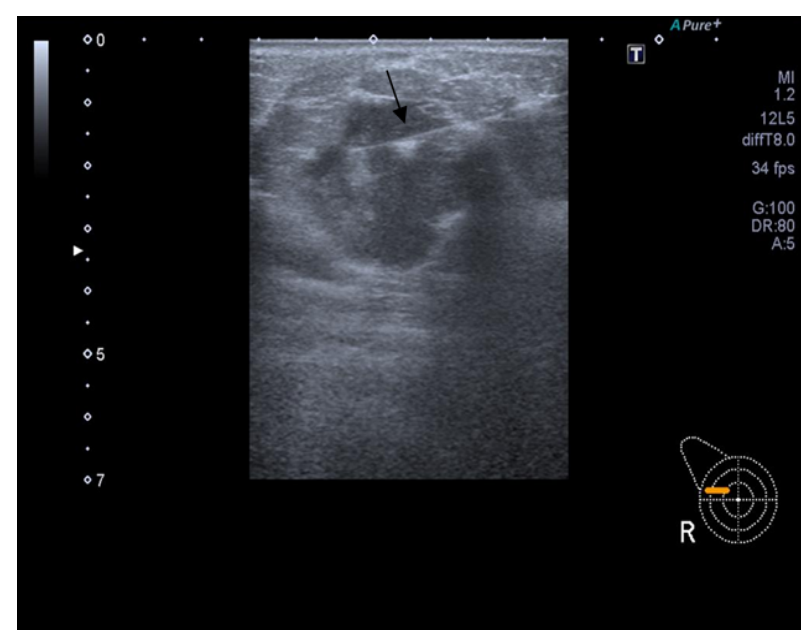

Figure 1. Ultrasound Guided Core Biopsy Needle within the Lesion (Arrow)

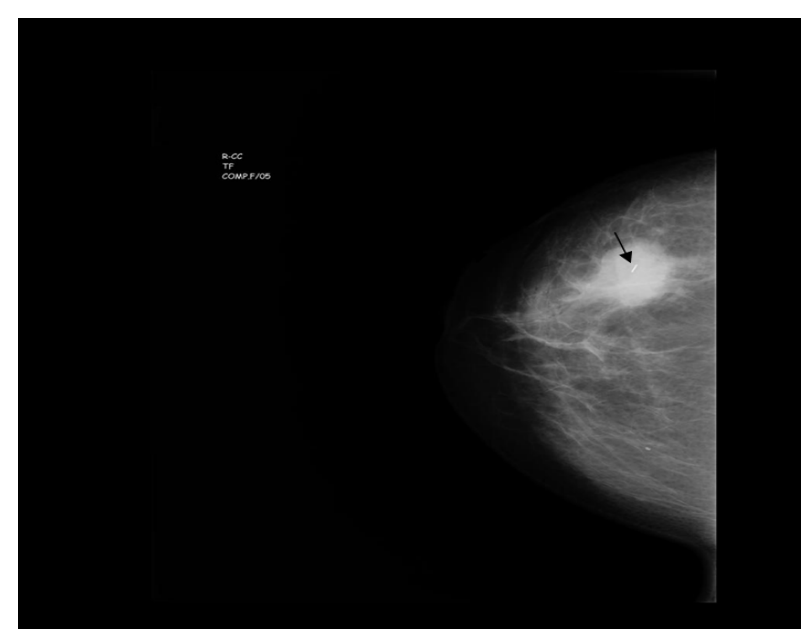

Figure 2. Craniocaudal View of Right Breast Showing Ligaclip within Center of Index Lesion (Arrow) specimen. There were no complications associated with clip placement such as pain, infection and hemorrhage. Out of 25 patients, 17 had Invasive Ductal Carcinoma (IDC) grade III, 5 had IDC II, 2 patients of ductal carcinoma in situ (DCIS) and 1 patient had metaplastic carcinoma grade III.

The total cost saving for 25 patients was PKR 202,500 (US\$ 2,025). The cost saving for individual patient was PKR 81,00 (US\$ 81).

\section{Discussion}

Neo-adjuvant chemotherapy (NAC) is now a standard step in treatment of locally advanced breast cancer when it is in early stage. NAC leads frequently to better surgical results by conserving the breast or making inoperable tumors excisable while lessening complications and improving cosmetic outcomes.

At our institution, clip placement prior to NAC is a standard procedure because one cannot predict the tumor response to NAC. In the past, cases were seen where the tumors showed complete radiological response both on mammography and ultrasound making localization of tumor bed impossible. Therefore, all patients who are to receive NAC undergo clip placement and are followed up by mammography and subsequent ultrasound to see tumor response. Magnetic Resonance Imaging is not performed

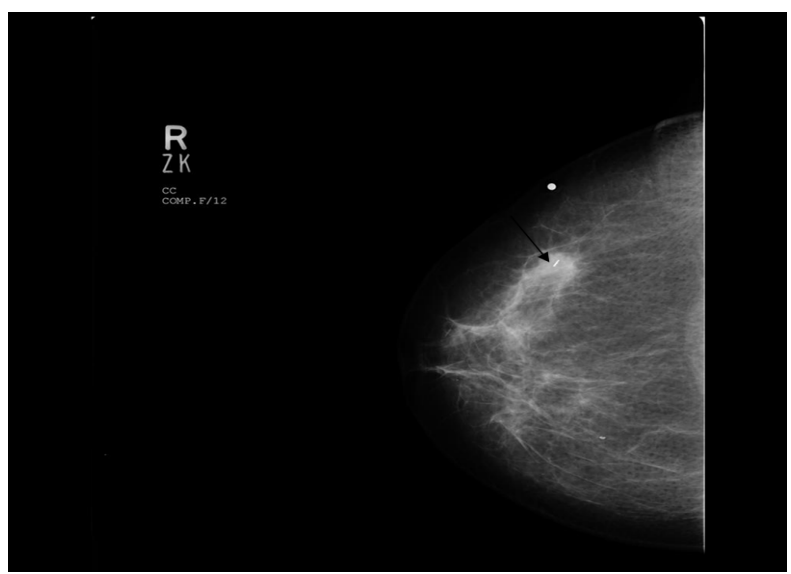

Figure 3. Craniocaudal View of Right Breast Showing Reduction in Size of Index Lesion when Compared with Figure 2 (Arrow)

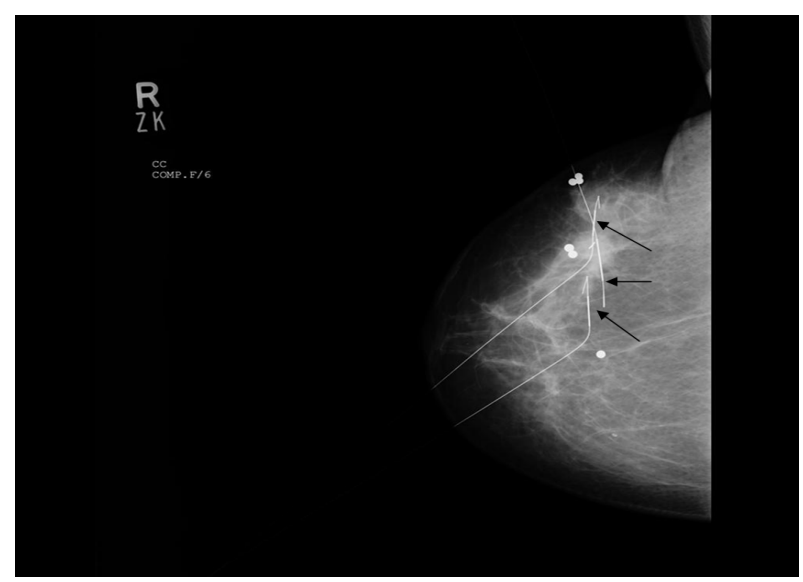

Figure 4. Craniocaudal View of Right Breast Showing Bracketing of Ligaclip with three Wires (Arrows) 


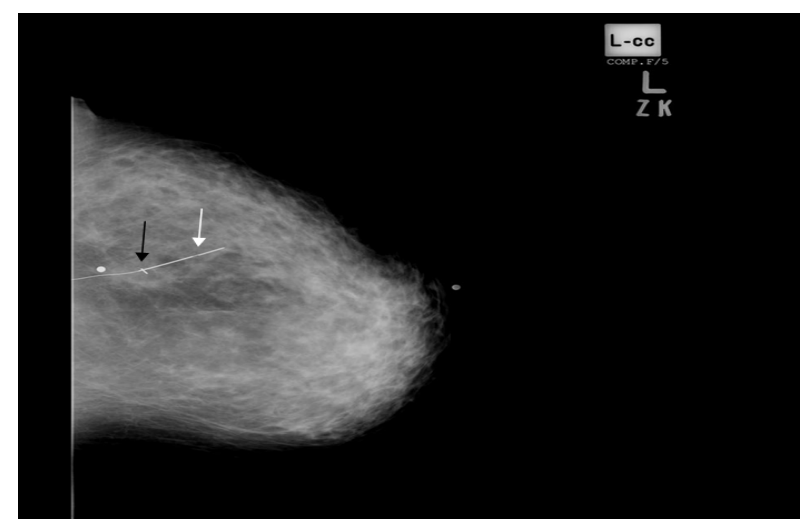

Figure 5. Craniocaudal View of Left Breast Showing Needle Localization of Ligaclip Prior to Excisional Biopsy (Black arrow =ligaclip, White arrow =localizing wire)

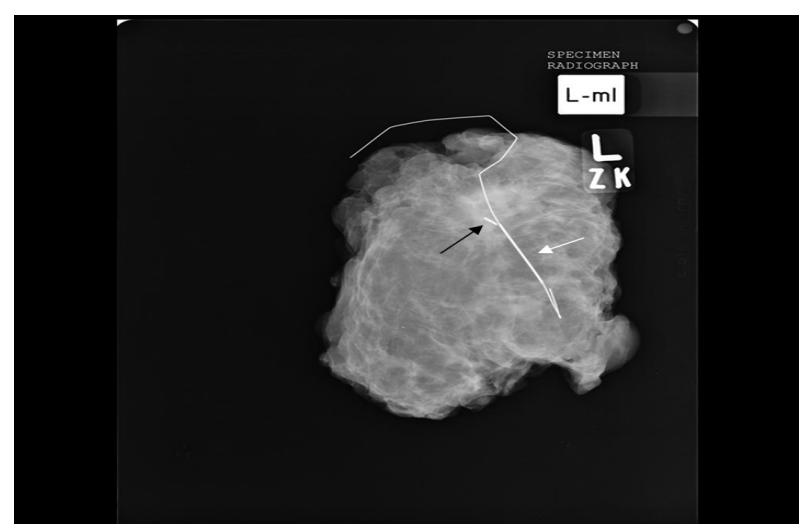

Figure 6. Magnified View of Excised Specimen Showing Ligaclip and Localizing Wire (Black arrow=Ligaclip, White arrow $=$ localizing wire)

routinely because being a third world country, patient affordability is a problem as all treatment costs are borne by the patients because of lack of health care system by government. The commercially available marker clips were not available in our institute around 5 years back when we started this practice. We, along with breast surgery team decided to use surgical ligature clips as an alternative to commercially available marker clips. Last year we were able to import the commercially available marker clips from abroad. These clips were expensive so there was a resistance from both the surgeon and patients regarding their insertion as they increased the cost of treatment further burdening the patient. When the patients were given choice of clip types almost all opted for surgical clip placement. The surgical clips placed had a cost saving of US\$ 81 per patient, and overall cost saving of US\$ 2,025 for 25 patients.

The study was conducted on hypothesis that surgical clips are cost effective, easy to place and are not associated with complications of clip migration and hemorrhage.

In our study, all clips were placed under ultrasound guidance in the same setting when a core biopsy of index lesion was performed. All clips were inserted using a $16 \mathrm{G}$ spinal needle after closing the surgical clip with an applicator and placing it into the lesion by using the stylet of the spinal needle. The correct clip placement was ascertained by ultrasound and doing two views of mammogram in orthogonal projections. The clip insertion using spinal needle was easy and safe as well as cost effective. In a similar study (Lee at al., 2005; Uematsu et al., 2007), the authors placed surgical clip by using automated gun for clip placement, but we used a spinal needle so we were able to precisely place it in the center of lesions, because in automated gun technique the placement is dependent on throw of needle. In another study (Youn et al., 2015), a semi-automated gun was used but in their study, core needle biopsy and clip insertion were done in two different sittings, as most of their patients had their core needle biopsy performed from another hospital. In contrast, we performed core needle biopsy of index lesion and any suspicious axillary lymph nodes along with clip placement in index lesion during the same visit for all patients as it is more convenient and a cost and time effective methodology. Clip migration can be a limitation of clip insertion especially in fatty breasts but the clip is inserted in the center of index lesion, thus minimizing clip migration due to higher resistance (Margolin et al., 2003). There was no clip migration at the time of clip insertion, post chemotherapy and in surgical specimen on mammography in all of our patients. The clips allowed easy identification of cancer on mammography and helped surgeons in explaining the findings to patients. Many studies in the past have concluded that placement of radiopaque markers are beneficial for tumor localization as well as for the assessment of tumor response to NAC, without disturbing radiologic evaluation of main lesion (Dash et al., 1999; Baron et al., 2000; Oh et al., 2007; Genson et al., 2007). We were able to evaluate tumor response post NAC and localize it with the help of mammography as a metallic density and on ultrasound as a small linear echogenic structure with or without shadowing. We did not perform MRI on our patients due to cost constraints. MRI is a better modality for assessing tumor response to chemotherapy, this has been reported in several studies but the metallic clip produces artifacts in form of signal void depending upon magnetic susceptibility, clip quality, size, shape, orientation, position and parameters used for MRI examination (Genson et al., 2007).

There are a few limitations of this study. The sample size was 25 , however with 25 patients in this study we still can conclude that there was significant cost saving per patient if we consider cost constraints for a self-paying patient in a third world country. The results for clip migration and complications cannot be generalized due to small sample; studies with larger sample size should be conducted to generalize the results on larger population groups. Another limitation is non-conductance of MR examination to see tumor response in our patients again because of cost constraints on patients. However we have evaluated tumor response to NAC by mammography and ultrasound and have obtained almost similar results to studies using mammography, ultrasound and MRI. However, in a study by (Genson et al., 2007), it is reported that artifacts caused by tissue marker clips can limit the sensitivity of MRI for detection and follow up of breast cancer.

In conclusion, we concluded from this study that ligaclip placement in patients with breast cancer 
undergoing NAC is a cost effective method for following response to NAC and is helpful in further surgical management post NAC. It is not associated with clip migration or significant complications related to clip placement but larger studies are needed to generalize our results.

\section{References}

Abdel-Razeq H, Marei L (2011). Current neoadjuvant treatment options for HER2-positive breast cancer. Biologics, 5, 87-94

Baron LF, Baron PL, Ackerman SJ, Durden DD, Pop TL Jr (2000). Sonographically guided clip placement facilitates localization of breast cancer after neoadjuvant chemotherapy. Am J Roentgenol, 174, 539-40

Dash N, Chafin S, Johnson RR, Contractor FM (1999). Usefulness of tissue marker clips in patients undergoing neoadjuvant chemotherapy for breast cancer. Am J Roentgenol, 173, 911-7

Ediken BS, Fornage BD, Bedi DG, et al (1999). US- guided implantation of metallic markers for permanent localization of the tumor bed in patients with breast cancer who undergo preoperative chemotherapy. Radiology, 213, 895-900

Genson CC, Blane CE, et al (2007). Effects on breast MRI of artifacts caused by metallic tissue marker clips. Am J Roentgenol, 188, 372-6

Kaufmann M, von Minckwitz G, Bear HD, et al (2007). Recommendations from an international expert panel on the use of neoadjuvant (primary) systemic treatment of operable breast cancer: new perspectives 2006. Ann Oncol, 18, 1927-34.

Kaufmann M, von Minckwitz G, Mamounas EP, et al (2012). Recommendations from an international consensus conference on the current status and future of neoadjuvant systemic therapy in primary breast cancer. Ann Surg Oncol, 19, 1508-16

Lee SY, Kook SH, Kwag HJ (2005). The results and usefulness of marker clip placement after ultrasound- guided mammotome excision of breast lesion. J Korean Radiol Soc, 52, 207-13

Lobbes MB, Prevos R, Smidt M, et al (2013). The role of magnetic resonance imaging in assessing residual disease and pathologic complete response in breast cancer paᄀtients receiving neoadjuvant chemotherapy: a systematic review. Insights Imaging, 4, 163-75

Margolin FR, Jacobs RP, Denny SR, Schrumpf JD (2003). Clip placement after sonographically guided percutaneous breast biopsy. Breast J, 9, 226-30

Oh JL, Nguyen G, Whitman GJ, et al (2007). Placement of radiopaque clips for tumor localization in patients un $\neg$ dergoing neoadjuvant chemotherapy and breast conservation therapy. Cancer, 110, 2420-7

Uematsu T (2007). Commercially available titanium clip placement following a sonographically guided core needle biopsy of breast. Breast J, 13, 624-6

Youn I, Choi SH, Kook SH, Choi YJ, et al (2015).Ultrasonographyguided surgical clip placement for tumor localization in patients undergoing neoadjuvant chemotherapy for breast cancer. J Breast Cancer, 18, 44-49. 